\title{
An E-education Framework Based on Semantic Web Agents
}

\author{
Ming Dong and Rong Rong \\ School of Information, Renmin University of China \\ 59 Zhongguancun Ave, Beijing, 100872, P.R.China \\ dongming@ruc.edu.cn, rongbaba@msn.com
}

\begin{abstract}
The booming web technology stimulates computer-based eeducation which is considered to be an appropriate lifelong education approach in this era due to the spatial and temporal convenience. In this paper we propose a service-oriented agent e-education framework based on the next generation web, viz. semantic web. Compared with the framework on which the e-education performs now, this new framework has many advanced properties due to the use of intelligent agents and is expected to perform an automated style for the future education.
\end{abstract}

\section{Introduction}

Nowadays, the lifelong education and re-training are considered as key aspects to both individuals and enterprises. Unfortunately, the traditional classroom education seems unsuitable today due to time, distance and cost limitations caused by modern intensive lifestyle [1,2]. Moreover, in this new era, learners are supposed to get the specific knowledge corresponding to their own needs. E-education seems to be a proper approach for achieving the objects above, for its abilities to overcome the temporal, spatial and learning style problems. In [3] the author predicated much of companies training would shift to e-learning or other web based activities in the future and by the year 2010 , the e-education market would reach $\$ 50$ billion. Eeducation allows teachers and students to finish the education activities in a virtual classroom via the booming development of the network technology. In this paper we propose an intelligent agents e-education framework based on the next generation web, the semantic web, to construct a better formed e-education system. This new framework makes education activities automated while optimizes the share of learning materials. So, it is supposed to be a promising framework for the future eeducation. 


\section{Preliminaries}

\subsection{Semantic Web}

E-education changes learning concepts in several aspects, it offers more personalized and adaptive learning style. Unfortunately, the current web based methods are not considered to be a suitable platform for implementing the e-education, because of many limitations, e.g. lack of intelligence. Semantic web which has higher capacities on information sharing, nonhuman autonomous implementing and communication supporting seems to be a proper platform to satisfy the requirement of e-education [4, 5].

In semantic web, contents are described by formal semantics. By means of this, resources on semantic web are much easier for machines to understand. Thus, an autonomous e-education system could be constructed well. The resources provider (teacher) prepares learning resources, and the consumer (student) describes his own situations and interests, his learning agent will search for learning materials of his own circumstance. This search is performed by semantic querying and navigation, which is considered more accurate compared to current technology.

In this paper, we propose an e-education framework on Semantic Web formed by intelligent agents. All the agents use commonly agreed service language. Thus, communication between agents is easy to achieve. Each agent has a specified function; it receives requests and matches them with semantic descriptions. At last, implements corresponding applications and acknowledges the request.

\subsection{Agents}

Agents are software-based autonomous systems made for specific functions to finish several tasks automatically in order to make the specific process much easier to users. Their functions could cover administrating process, fetching matched resources, triggering specified events, formalizing specifications etc. With the help of intelligent agents the whole process is transparent to learners and teachers.

In our proposed framework, intelligent agents are introduced to finish the granulated model functions. The learners/teachers only need to describe their own demands then the corresponding agents will finish the work and transport information to the correct applications, each agent also could call other agents for collaboration, e.g. these is no matched learning material on one server agent (will be introduced later). So, this Server agent calls other server agents to match the materials and transport them back.

\section{Proposed Framework}

\subsection{Overview}

In the following context, we will present our framework in detail. In the proposed framework, each user (students and teachers) will have a personal agent. These personal agents have their own knowledge bases which contain the formalized 
commands, interface protocols and user's profiles besides. Each personal agent receives demands and data from the user and generates commands corresponding to user's profiles and demand. Each server agent receives commands from other agents and sends feedbacks back to the enquirer. In [9, 10], similar frameworks are presented; but we supposed our framework is more efficient and convenient to build larger e-education systems due to two reasons. Firstly, in our framework, every agent generates formalized commands which could be recognized by other agents and every agent has the ability to communicate to other agents; these properties are highly advantaged to form an active e-education environment without adding so many burdens to the server. Secondly, server agents could be made heuristically; all these server agents could be distributed all around the world, we could use cascaded enquiry to get the corresponding materials. These cascaded server agents are much easier to maintain and modify, but still preserving easy entrance property. With the help of semantic web, all learning materials could be well annotated and shared; then an internet-liked e-education system will be built.

\subsection{Framework Layout}

Fig.1 describes the top-level view of our proposed framework. In this framework, we use the service-oriented model [7] to perform education activities. In service-oriented model, students control whole process, they describe their own demands and offer other relevant information (their situation etc.). Teachers provide learning materials and other educational services related to their courses under the specific description rules(such as LOM, ARIADNE, IMS, etc.). Student agents receive demands from students, finish their own functions and call Server agents to provide corresponding services. Sever agents receive orders sent by student agents and match descriptions with its own knowledge base then generate the relevant services and send these information back to students/teachers/other agents or trigger the corresponding applications.

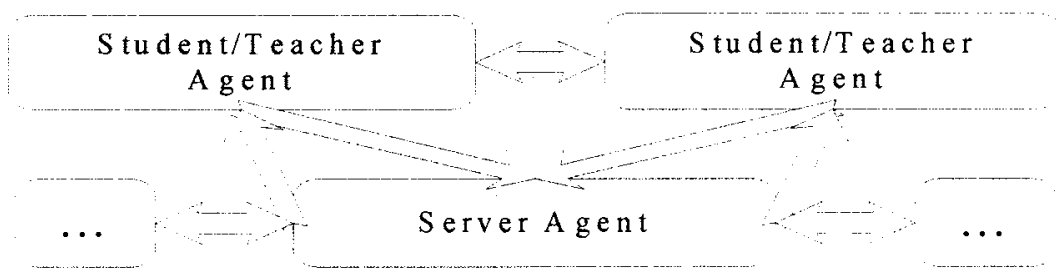

Fig. 1. Top level of the e-education framework

\subsection{Infrastructure}

\section{The Students/Teacher Agents}

In our framework, the students/teacher agent is composed of three main function blocks. Namely, application trigger, command generator and interface manager. Command generator is used to generate the required description which depicts the demands and situations of current user. Interface Manager contributes to formalization of the communication with sever agents. Both Command Generator 
and Interface Manager search the knowledge base to match the relevant items and then generate results. Application trigger receives information from other agents and triggers corresponding applications to finish the e-education activities. E.g. if the sever agent decides to transport an educational video back to the student agent, the application trigger will accept this video and then initialize the video player.

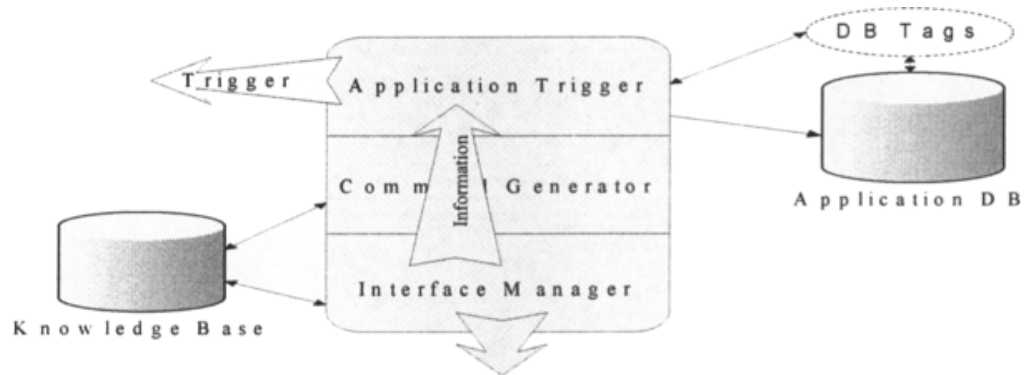

Fig. 2. The infrastructure of the Students/Teacher Agents

\section{The Server Agents}

The structure of server agent is represented in Fig.3. There are several sub-agents in this system to perform specific functions on the semantic web; all the sub-agents could communicate with other sever agents to derive relative information/resources.

In server agent, the first layer is portal. This portal waits for requests from other agents. After receiving a request from student, teacher or other agents, user's status will be checked by authentification\&recognition process. Then different rights will be allocated for the following procedures. Meanwhile system reads the user's information from DB. E.g., for students the information could be: research directions, learning history, etc.
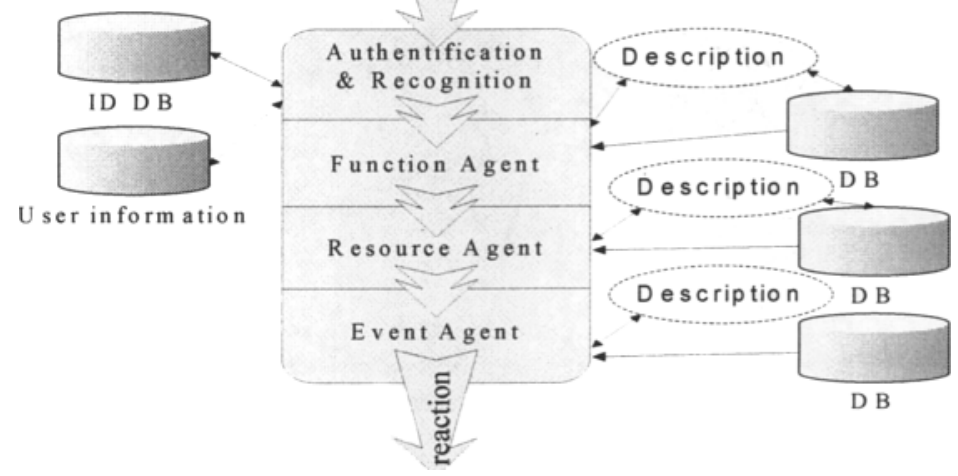

Fig. 3. The infrastructure of the Server Agents 
After decoding received orders, server agent would generate corresponding responds. In the function agent layer, function agent matches the needed function with descriptions from the user. E.g. look at fig.4, we give a demonstration of the function matching. The inspiration is derived from[1].

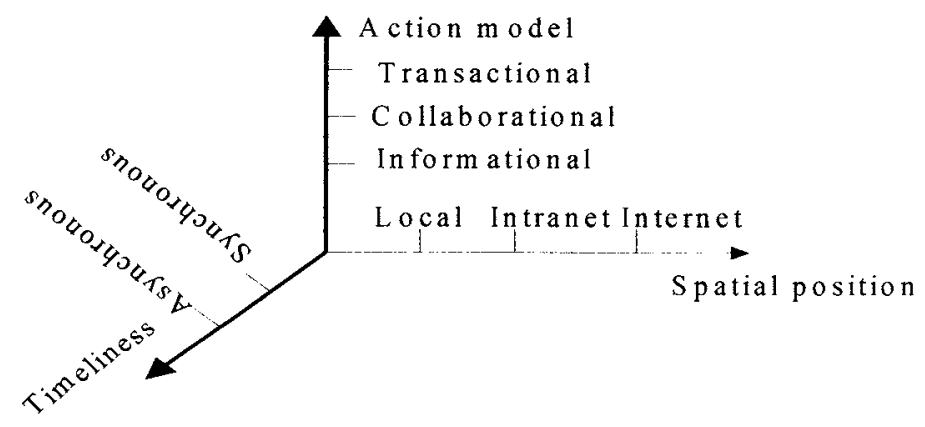

Fig. 4. Function Matching

Function space is divided into small cells; the granularity depends on agent designers. In this demonstration, the space is divided into 18 cells; agents categorize every function into these cells and match the functions with the user's status. E.g. a student wants to take a net-meeting with his teacher, if this student is not a full-time student in this university, then this demand will be classified into "Internet, Collaborational, Synchronous' cell; after this the agent could match the demand with services belong to this cell.

After selecting correct function, resource agent and event agent will select correct resources to users and generate relative service events corresponds to user's preferred behaviors and results from pre-steps. After these steps the server agent will initial the selected events. These events could be a learning resource delivery from/to a teacher/student, triggering of connection between a teacher and a student, visiting another server agent for getting relevant resources, etc. Finally, after acknowledging the request, the sever agent will finish its jobs and student/teacher agent will carry on their further jobs depends on the resources and instructions received from the server agent.

\section{Conclusion}

We proposed an e-education framework based on semantic web agent. This framework is a service-oriented one. So, students are in the center of the education, all the agents and teachers are passive and collaborational, they only offer the services to students. This property maximizes the benefits of students; they could make their own plan corresponding to themselves. The semantic agent approach is a quite advanced method to perform the e-education based on the next generation web. Semantic standard could ultimately share and get the correct resources to a certain circumstance; meanwhile agent method could get more flexibility, modularity and 
customization. In all, it is an effective method to perform e-education with the emerging of new web technology.

\section{References}

1.L.F. Motiwalla, "An e-education framework for training in the next generationenterprises", Research Challenges, 2000. Proceedings. Academia/Industry Working Conference , 139-144(2000).

2.T.F. Stafford, "Understanding motivations for Internet use in distance education", Education, IEEE Transaction, 48(2),301-306 (2005).

3.K. Levis, "The Business of E-learning: A Revolution in Training and Education Markets". http://www.hrmguide.net/usa/hrd/e-learning_survey.htm.

4.http://www.aifb.uni-karlsruhe.de/WBS/Publ/2001/WebNet_Istsstrst_2001.pdf.

5.T. Anderson and D. Whitelock, "The educational semantic Web: visioning and racticing the future of education", Journal of Interactive Media and Education , 1, 2004.

6.N. Hussain and M.K. Khan, "Service-Oriented E-Learning Architecture Using Web Service-Based Intelligent Agents", Information and Communication Technologies, 2005. ICICT 2005. First International Conference, 137-143(2005).

7.R.D. Andreev and N.V. Troyanova, "E-learning Design: An Integrated Agent-Grid Service Architecture", Modern Computing, 2006. JVA '06. IEEE John Vincent Atanasoff 2006 International Symposium, 208-213(2006).

8.Z. Abbas, M. Umer, M. Odeh, R. McClatchey, A. Ali and A. Farooq, "A semantic grid-based e-learning framework (SELF)", Cluster Computing and the Grid, 2005. CCGrid 2005. IEEE International Symposium, 1, 11-18(2005).

9.P. Nilas, N. , Nilas and S. Mitatha, "A Dynamic Associative E-Learning Model based on a Spreading Activation Network", Electrical and Computer Engineering, Canadian Conference ,2472-2475(2006).

10. Y. Shang, H.C. Shi and S.S. Chen, "An Intelligent Distributed Environment for Active Learning", Journal of Educational Resources in Computing, 2001.

11. R. Koper, "Use of the Semantic Web to Solve Some Basic Problems in Education", Journal of Interactive Media in Education, 2004. 